\title{
Topical Corticosteroid Abuse in Dermatology
}

\author{
${ }^{1 *}$ Anup KMishra, ${ }^{2}$ Devesh Saraswat, \\ ${ }^{1}$ Assistant Professor, ${ }^{2}$ Professor, and Head \\ Department of Dermatology, SS Medical College, Rewa, Madhya Pradesh
}

\begin{abstract}
Topical corticosteroids are the most widely used therapeutic agents in modern dermatologic therapy. The increasing frequency of use of topical steroids has led to the detection of a number of adverse effects and is dependent upon chemical nature of the drug, the vehicle, and the location of its application. The common adverse effects on face are erythema, telangiectasia, xerosis, photosensitivity and rebound phenomenon. The improper advertisement by manufacturers, availability of steroids without prescription, improper prescribing by doctors, has resulted in the rapid emergence of topical steroid abuse.
\end{abstract}

Aims and Objectives: To analyse the magnitude and adverse effects of topical steroids abuse in dermatology.

Materials \& Methods: This is a prospective questionnaire type study conducted in outpatient clinic of the Department of Dermatology in a tertiary hospital. A total of 200 patients of any age and of both sexes are recruited consecutively. A questionnaire eliciting demographic variables, characteristics of topical steroid use, prescription source, duration of application and adverse effects is administered to all eligible patient. Patients not consenting to answer the questionnaire or patients with co-morbidities that could cause similar changes to topical steroid side effects like Cushing's syndrome, thyroid disorders, polycystic ovaries were excluded. Statistical analysis was done using SPSS ver. 21.

Results: The most common age group in which these side effects were observed is 21-30 years. Clobetasol propionate, betamethasone valerate and mometasonefuroate were the commonly used steroid by patients. It was found that $29 \%$ of patients using topical steroid were recommended by friend or pharmacist and $71 \%$ by a medical practitioner. Topical steroid abuse was seen in all 58 patients (100\%) prescribed by friend or pharmacist and 58 out of 142 patients (40.84\%) prescribed by medical practitioner. Tinea incognito and striae were the common side effects and were seen more commonly in non-dermatologists group.

Conclusion: This study reveals that problem of topical steroid misuse is common in our country. The prevalence of side effects is significantly higher among the potent steroid users. The prevalence of side effects is significantly higher among patients of non-dermatologists $(p<.05)$ indicating that the schedule and explanation of guidelines for correct application of steroids is a drawback among non-dermatologists.

Recommendation: Education of the general public through special media programmes and the continuing medical education programme for medical and paramedical personnel are probably the most important steps that could be undertaken to reduce this problem.

Keywords: Corticosteroid Abuse, Dermatology, Topical Application

\section{Introduction}

Topical corticosteroids have made a dramatic contribution to dermatology since the introduction of "compound F" or hydrocortisone in 1952 and have become the mainstay of dermatologic treatment of a wide range of inflammatory and non-infectious conditions [1]. Topical corticosteroids are today among the most commonly prescribed medications in dermatology clinics. The clinical effects are mediated by their antiinflammatory, vasoconstrictive, anti-proliferative and immunosuppressive properties [2].They are highly efficacious drugs which are used for the treatment of varied autoimmune and inflammatory dermatological conditions [2].

In India, the annual sales figure of TCs was 14 billion rupees in 2013, which accounts for almost $82 \%$ of total dermatological product sale in the country [3] Prescription of TC has become ubiquitous with dermatologists and concerns have also been raised regarding its misuse for non-labeled indications. As per the information available on the Central Drugs Standard Control Organization (CDSCO) website regarding approved dermatological indications of TC (although indications are not mentioned for all the TC molecules), its off-label use seems to be a common clinical practice in India [4,5]. However, the more serious concern is its inappropriate use in symptomatic treatment for varied dermatological disorders like acne, primary bacterial and fungal infections, undiagnosed skin rash and as fairness cream by non-registered practitioners or on the advice of pharmacist at chemist shops [6-8].

Most of these medications are sold without any prescription or patients are easily able to procure these steroid medications on a single prescription repeatedly from a local pharmacist. This leads to increased 
frequency of adverse effects and often dependence on these medications. To make the problem worse India has very few qualified dermatologists to prescribe these drugs rationally.

Not only developing countries like India and Africa but even developed countries like United States of America is facing this problem. Urgent steps are therefore recommended to curb the root of this problem at the earliest. The present study was thus conducted to analyze the magnitude and adverse effects of topical steroids abuse on the body in dermatology outpatient department of a tertiary hospital and to recognize the offending topical steroids in order of frequency.

\section{Materials \& Methods}

This is a prospective questionnaire type study conducted in outpatient clinic of the Department of Dermatology, SS Medical College and hospital, Rewa, MP, during the period May 2015 to May 2016. A total of 200 patients irrespective of age and sex were recruited consecutively. A questionnaire eliciting demographic variables, characteristics of topical steroid use, prescription source, duration of application and adverse effects was administered to all eligible patients.

\section{Inclusion criteria}

All the patients who had used topical steroid over the body for certain skin problems for more than 1 month and presented in Dermatology outpatient department are included. Wrong indication, undiagnosed dermatoses, inappropriate potency or more than 1 month use after the last consultation are criteria used to define inappropriate or unjustifiable use. Adverse effects following steroid abuse were also studied.

\section{Exclusion criteria}

Patients not consenting to answer the questionnaire or patients with co-morbidities that could cause similar changes to topical steroid side effects like Cushing's syndrome, thyroid disorders, polycystic ovaries were excluded.

\section{Statistical Analysis}

All the data was collected and entered in Microsoft excel sheet 2013 and statistical analysis was done using SPSS ver. 21.0.

\section{Results}

Mean age of study subjects was 32.3 years with slight male predominance (57\%). Out of 200 patients, 108 (54\%) patients were recommended topical steroid by a dermatologist while 92 (46\%) by nondermatologists. Of which $10.5 \%$ and $18.5 \%$ were prescribed steroid by either a friend or a pharmacist (Table 1). Majority of the patients i.e. 57 (28.5\%) had come with fungal infection followed by $21(10.5 \%)$ cases of lichen Planus. Vitiligo, Melasma and Psoriasis were found in 14 (7\%), 13 (6.5\%), 13 (6.5\%) cases respectively (Table 2). Adverse effects were observed in 85 (42.5\%) patients out of 200. Three patients had more than one side effect. Most common side effect was tinea incognito followed by striae and telangiectasia (Table 3). Most common topical steroid used by patients was clobetasol propionate (30.5\%) (Table 4). Out of 200 patients 139 $(69.5 \%)$ used pure steroid cream while $61(30.5 \%)$ used steroid cream in combination with either antifungal or antibacterial or both. Patients using cream based applications were $147(73.5 \%)$ while $52(26 \%)$ of them used ointment based applications. Only $1(0.5 \%)$ patient used gel preparation. Frequency of applications was twice daily in $148(74 \%)$ patients and once a day in 52(26\%) patients (Non tabulated). There were significantly higher chances of side effects in patients if the prescription is from a non-dermatologist and on application of potent steroids $(\mathrm{p}<0.05)($ Table 5$)$.

\section{Discussion}

Topical corticosteroids were first introduced for use in 1951 and have now become the most commonly used drugs by dermatologists. Their effect in suppressing inflammatory dermatoses makes them an indispensible therapeutic tool. However, they are considered as - double edged swordll drugs i.e. invaluable but need careful handling by the provider and the recipient for safe and effective use [9]. Since their invention, uncontrolled use (abuse) of steroid medications has lead to many different adverse reactions. Multiple pathways including rebound vasodilatation and proinflammatory cytokine release have been proposed as the mechanism for such adverse reactions [10].

Our results clearly show that misuse of topical corticosteroids is common in our country. This is evident by number of patients who visited our department with side-effects of these drugs. Even then, adverse effects and safety of topical corticosteroids are neglected in the medical literature. The root of problem is that patients are easily able to get these topical steroid medications on a single prescription repeatedly from a local pharmacist. This leads to occurrence of sideeffects and often dependence on these medications. In our country it 
is a major problem because patients are able to procure a drug without a valid prescription from a medical practitioner. The problem is worsened when these steroid containing creams are freely advertised on television, thus it is not only a medical but also a social problem.

The patient might initially start using these creams for some undiagnosed dermatoses which are often prescribed by their friends and relatives. At first the vasoconstrictive and anti-inflammatory effects of the steroids result in clearance of the primary dermatoses but on continuation of the application, they develop rashes, and on stopping the drug, there is recurrence of rashes i.e. rebound phenomenon. So, to prevent this effect, the patient resumes the use of steroid creams.

The reason for consulting a doctor by most of them is when they found their skin problem no longer responds to the so called "magical effect" of the cream. Persistent use by then leads toepidermal atrophy, degeneration of dermal structure and collagen deterioration after several months. Continued or overuse of steroids can result in thinning of the skin as well as dependency on the steroid [10]. The appearance of these side effects depend on the potency of steroid, duration of use and the site of application.

In present study a total of 200 patients were included. Wrong indication, undiagnosed dermatoses, inappropriate potency or more than 1 month use after the last consultation were criteria used to define inappropriate or unjustifiable use. Age range of patients in present study was from 2 years to 74 years with a mean age of 32.3 years. Out of a total of 200 patients, $114(57 \%)$ were male and $86(43 \%)$ were female. Eighty five of these 200 patients developed adverse effects from the use of steroids. The most common age group in which these side effects were observed is $21-30(38.82 \%)$ years. This is probably because patients in this age group are overcautious with their appearance and with different skin conditions. Similar studies done in India show most common age for topical steroid abuse as 20-30 yearsand in Iraq it was 10-19 years [11,12].

Dermatophytic (28.5\%) group of infections was the most common indication for which topical steroids were prescribed followed by lichen planus (11.5\%). Vitiligo and Melasma were other common indications for which steroids were used. In other similar studies from India and Iraq, topical steroids were most commonly used as a skin lightening cream [11,12].

Adverse effects were observed in $85(42.5 \%)$ patients. Commonest side effect which was seen in our study was Tinea incognito (19.5\%) followed by striae (7\%) and hypopigmentation (6.5\%). Similar studies done by Saraswat et al and Al-Dhalimi et al had acne as the most common side effect as topical steroids were commonly used as a fairness cream $[11,12]$.

Clobetasol propionate was used in $30.5 \%$ of patients while Betamethasone valerate in $28.5 \%$ of the total cases. Mometasonefuroate was the other common steroid used by patients. Betamil GM and Betnovate were the popular brand names which were used by patients. Similar study done in Iraq also showed clobetasol propionate $(42.1 \%)$ as the most commonly used steroid while betamethasone valerate $(26.4 \%)$ was the second most commonly used steroid [12].In another study done by Saraswat et al. betamethasonevalerate $(50.1 \%)$ was the most commonly used topical steroid [11].

Most of the patients used topical steroids for a short duration of time (1-2 months) and had come to the Dermatology outpatient department because of their adverse effects. Source of prescription was studied and it was found that $29 \%$ of patients using topical steroid were recommended by friend or pharmacist and $71 \%$ by a medical practitioner. Out of these $71 \%$, most of the prescriptions were by dermatologists (54\%) followed by physician $(16 \%)$ and general practitioner $(1 \%)$. According to our criteria used to define inappropriate or unjustifiable use, topical steroid abuse was seen in all 58 patients (100\%) prescribed by friend or pharmacist and 58 out of 142 patients $(40.84 \%$ ) prescribed by medical practitioner. A study done on topical steroid abuse on face by Saraswat et al showed that $59.3 \%$ of the total patients had used topical steroids on recommendation without a valid medical prescription [11].

The potency of steroids was studied and according to the classification of topical steroidsused, it was seen that although prescription by both non dermatologists and dermatologists were for products in potent group but adverse effects were more often seen in patients of non-dermatologists. This was due to lack of knowledge of potency of topical steroids by non-dermatologist group leading to their prescription in conditions in which it was either not justified or a lesser potent steroid could have been given, resulting in increased misuse and therefore adverse effects in these patients. Similar findings were observed by Al Dhalimi et al. in Iraq where potent steroids were prescribed by both the groups [12].

This study reveals that problem of topical steroid misuse is common in our country. In India free availability of these medicines has allowed many of these brands to become householdnames. Patients are unaware of risks posed by these drugs and continue to use them for long periods before seeking help from dermatologists. Improper advertisement by manufacturers, availability of steroids without prescription, improper prescribing by non-dermatologist doctors and lack of proper knowledge about these drugs has resulted in misuse of these steroid medications on a large scale [13]. 


\section{Conclusion \& Recommendations}

From our study it was concluded that topical steroid abuse is prevalent in our country. Rapidity of symptomatic relief is one of the major reasons for its misuse. The problem is worsened when patient is easily able to get these medications even without a proper prescription. In our study it was found that steroid abuse was most common in younger age group. Thus, easy availability of topical steroid, poor access ofpatients to dermatologists and lack of knowledge of the non-dermatologists make steroid abuse a common problem in India. As indicated by the data in our study, this problem is already significant and urgent steps should be taken to control this problem. Besides, it is a multiphasic problem that needs the cooperation of different sectors in the community to overcome it. Education of the general public through special media programmes and the introduction of continuing medical education programme for medical and paramedical personnel are probably the most important steps that could be undertaken to reduce this problem

\section{Bibliography}

[1]. Sulzberger MB, Witten VH. The effect of topically applied compound F in selected dermatoses. J Invest Dermatol 1952; 19:101-2.

[2]. Valencia IC, Kerdel FA. Topical glucocorticoids. In: Fitzpatrick T, editor. Dermatology in General Medicine. 5 th ed. New York: McGraw-Hill; 1999. p. 2713-7.

[3]. Verma SB. Sales, status, prescriptions and regulatory problems with topical steroids in India. Indian J DermatolVenereolLeprol 2014;80:201-3.

[4]. 4. Fixed Dose Combinations approved By DCG (I) since 1961 till July, 2014. Central Drugs Standard Control Organization, India.

[5]. Approval Status of New Drug during 1971-1998. Central Drugs Standard Control Organization, India. Available from: http://cdsco.nic.in/ writereaddata/1971-98.doc. [Last accessed on 2015 May 28].

[6]. Saraswat A, Lahiri K, Chatterjee M, Barua S, Coondoo A, Mittal A, et al. Topical corticosteroid abuse on the face: A prospective, multicenter study of dermatology outpatients. Indian J DermatolVenereolLeprol 2011;77:160-6.

[7]. Ambika H, VinodCS, Yadalla H, NithyaR, BabuAR. Topical corticosteroids abuse on face: A prospective, study on outpatients of dermatology. Our Dermatol Online 2014;5:5-8.

[8]. Rathod SS, Motghare VM, Deshmukh VS, Deshpande RP, Bhamare CG, Patil JR. Prescribing practices of topical corticosteroids in the outpatient dermatology department of a rural tertiary care teaching hospital. Indian J Dermatol 2013;58:342-5.

[9]. Saraswat A. Topical corticosteroid use in children: Adverse effects and how to minimise them. Indian J DermatolVenereol 2010;76:225-28.

[10]. Bhat YJ, Manzoor S, Qayoom S. Steroid - induced rosacea: A clinical study of 200 patients. Indian J Dermatol, 2011;56:30-32.

[11]. Saraswat A, Lahiri K, Chatterjee M, Barua S, Coondoo A, Mittal A, et al. Topical corticosteroid abuse on the face: A prospective, multicenter study of dermatology outpatients. Indian J DermatVenerolLeprol 2011;77:160-66.

[12]. Al-Dhalimi MA and Aljawahiry N. Misuse of topical corticosteroids: A clinical study in an Iraqi hospital. Eastern Mediterranean Health Journal 2006;12:847-52.

[13]. Lu H, Xiao T, Lu B, Dong D, Yu D, Wei H, Chen HD. Facial corticosteroid addictive dermatitis in Guiyang City, China. ClinExpDermatol 2010;35:618-21.

Tables

Table 1. Baseline Variables in the study group

\begin{tabular}{|l|c|}
\hline Variables & Mean (SD)/ n(\%) \\
\hline Age (years) & $32.3(5.6)$ \\
\hline Male & $114(57 \%)$ \\
\hline Female & $86(43 \%)$ \\
\hline$</=1$ month use & $164(82 \%)$ \\
\hline$>$ 1 month use & $36(18 \%)$ \\
\hline Dermatologist & $108(54 \%)$ \\
\hline Physician & $32(16 \%)$ \\
\hline General Practitioner & $2(1 \%)$ \\
\hline Pharmacist & $37(18.5 \%)$ \\
\hline Friend & $21(10.5 \%)$ \\
\hline
\end{tabular}

Table 2. Distribution based on Clinical Indication of steroid use

\begin{tabular}{|l|c|c|}
\hline Indication & Frequency (n-200) & Percent \\
\hline Dermatophytic Infections & 57 & $28.5 \%$ \\
\hline Lichen Planus & 23 & $11.5 \%$ \\
\hline Vitiligo & 12 & $6.0 \%$ \\
\hline Melasma & 13 & $6.5 \%$ \\
\hline Psoriasis & 13 & $6.5 \%$ \\
\hline Atopic Dermatitis & 10 & $5.0 \%$ \\
\hline Acne & 9 & $4.5 \%$ \\
\hline Alopecia Areata & 11 & $5.5 \%$ \\
\hline P. Rosea & 10 & $5.0 \%$ \\
\hline P. Alba & 12 & $6.0 \%$ \\
\hline Polymorphic Light eruptions & 11 & $5.5 \%$ \\
\hline Others & 34 & $17.0 \%$ \\
\hline
\end{tabular}


Table 3. Distribution based on type of adverse effects

\begin{tabular}{|l|c|c|}
\hline Adverse Effects & Frequency (n-88) & Percent \\
\hline Tinea Incognito & 39 & $44.3 \%$ \\
\hline Striae & 14 & $15.9 \%$ \\
\hline Hypopigmentation & 12 & $13.6 \%$ \\
\hline Telangiectasia & 10 & $11.4 \%$ \\
\hline Acneform Eruptions & 10 & $11.4 \%$ \\
\hline Milia & 2 & $2.3 \%$ \\
\hline Perioral Dermatitis & 1 & $1.1 \%$ \\
\hline
\end{tabular}

Table 4. Distribution based on type of topical steroid used

\begin{tabular}{|l|c|c|}
\hline Drug Used & Frequency (n-200) & Percent \\
\hline Clobetasol & 61 & $69.3 \%$ \\
\hline Betmethasone & 57 & $64.8 \%$ \\
\hline Mometasone & 28 & $31.8 \%$ \\
\hline Fluticasone & 20 & $22.7 \%$ \\
\hline Fluocinolone & 10 & $11.4 \%$ \\
\hline Halobetasol & 8 & $9.1 \%$ \\
\hline Beclomethasone & 10 & $11.4 \%$ \\
\hline Desonide & 3 & $3.4 \%$ \\
\hline Others & 3 & $3.4 \%$ \\
\hline
\end{tabular}

Table 5. Association of Adverse events with type of prescription and potency of topical steroid used

\begin{tabular}{|l|c|c|c|}
\hline \multirow{2}{*}{\multicolumn{1}{|c|}{ Variables }} & \multicolumn{2}{c|}{ Adverse Event } & \multirow{2}{*}{ p- value } \\
\cline { 2 - 3 } & Yes & No & \multirow{2}{*}{$<0.05$} \\
\hline Non Dermatologist Prescription (n-92) & 71 & 21 & \multirow{2}{*}{$<0.05$} \\
\hline Dermatologist Prescription (n-108) & 14 & 94 & \\
\hline Potent Steroid (n-109) & 55 & 54 & \\
\hline Non potentSteroid (n-91) & 30 & 61 & \\
\hline
\end{tabular}

